\title{
Management of Elderly Patients with Diabetes Mellitus from Patient-Centered Medicine
}

\author{
Hiroshi Bando, MD, $P h D^{1,2^{*}}$ \\ ${ }^{1}$ Medical Research, Tokushima University, Japan \\ 2Japan Low Carbohydrate Diet Promotion Association (JLCDPA), Japan
}

\begin{abstract}
Diabetes mellitus has been an increasingly important health problem worldwide. In the case of elderly diabetic patients, adequate control of not only glucose variability but also physical, psychological, social, nutritional management would be crucial. Recent important topics include frailty, sarcopenia and locomotive syndrome. From mentioned above, elderly diabetics need patient-centered medicine with bio-psycho-social, leading to satisfactory and significant life.
\end{abstract}

\section{Keywords}

Low carbohydrate diet, Mini nutritional assessment, Type 2 diabetes mellitus, Patient-oriented research, Patientcentered medicine

\section{Abbreviation \\ LCD: Low Carbohydrate Diet; MNA: Mini Nutritional Assessment; T2DM: Type 2 Diabetes Mellitus; POR: Patient-Oriented Research; PCM: Patient-Centered Medicine}

Diabetes mellitus has become more important health problem across the world. Among them, it is not simply a medical problem with excess calories and obesity, but various differences and issues are found in several situation. Even if the value of $\mathrm{HbA} 1 \mathrm{c}$ is same, the guidance for standard regular diet is not the same between for the younger group and the elderly group.

From clinical research, evidence-based medicine (EBM) has been known, and patient-oriented research (POR) has been also important as the fundamental concept in ordinary medical practice [1]. Furthermore, patient-centered medicine (PCM) has been in focus as the basal philosophy of medical practice. For the integration of these aspects, POR and PCM have been expected to exist in an appropriate balance.

The author and colleagues have been conducting clinical research on calorie restriction (CR) and low carbohydrate diet (LCD) for Type 2 diabetic patients (T2DM) for long years [2]. Using the meal of $C R$, we have analyzed the response of blood sugar and insulin to $70 \mathrm{~g}$ of carbohydrate intake which is meal tolerance test (MTT) [3]. MTT has been useful and beneficial for speculating the response of glucose and insulin. As regard to LCD, we have analyzed the glucose variability and $M$ value, which could be improved in a short period by application of LCD. From our clinical practice and research, various related factors have been involved in the pathogenesis and management for diabetes in the elderly.

For diabetic patients, proper nutrition is fundamental to self-care. Its fundamental nutrition guidelines has been common regardless of the age [4]. However, various problems are found in the case of elderly diabetics [5]. Physical problems would include dental impairment, dysphagia, difficulties of taste and smell, gastrointestinal dysfunction and others. From social points of view, some difficulties are observed in going out for shopping, buying food, preparing meals, and leading health-related quality of life. Furthermore, there are possibly influencing diseases such as depression, dementia, mild cognitive impairment $(\mathrm{MCl})$, anorexia nervosa, and so on [6].

Unlike young diabetics, elderly diabetics are important to be assessed whether they have any nutrition problems or not. For that purpose, we can use short nutritional assessment questionnaire (SNAQ) and mini-nutrition evaluation mini nutritional assessment (MNA) in simple and easy way [7].

Previous reports have shown that early screening may

*Corresponding author: Hiroshi Bando, MD, PhD, FACP, Medical Research, Tokushima University, Nakashowa 1-61, Tokushima 770-0943, Japan, Tel: +81-90-3187-2485

Accepted: July 24, 2019

Published online: July 26, 2019

Citation: Bando H (2019) Management of Elderly Patients with Diabetes Mellitus from Patient-Centered Medicine. Insights Biomed Res 3(1):49-51 
be necessary, especially for elderly patients with nutritional problems [8]. They include home care residents and patients who were admitted due to acute change. These nutritional assessment can prevent future possible complications. When these evaluation is not conducted before, several problems may occur later, such as various complications, long and frequent hospitalization leading to unnecessary medical expenses [9].

When diabetic patient is more than 65-years-old associated with frailty or locomotive syndrome, adequate meals including enough amount of protein and calories are crucial. According to previous investigations, consuming protein-rich and high energy meal can bring the metabolic status with preventing malnutrition risk and weight loss. Elderly people show less protein intake which is necessary for a day as $0.8 \mathrm{~g} / \mathrm{kg}$.

Research group of PROT-AGE recommends more amount of taking protein per day, which are 1.0-1.2 g/ kg body weight a day to healthy elder person, and $1.2-1.5 \mathrm{~g} / \mathrm{kg}$ body weight a day to elder patients having chronic and/or acute diseased states. Furthermore, severe patients with sarcopenia and cachectic elder patients are recommended to take protein more than $1.5 \mathrm{~g} / \mathrm{kg}$ a day by the nutrition expert professionals [10]. On the other hand, consistent beneficial results were found by giving specific supplemental nutrients, such as creatine, protein supplements and branched-chain amino acids (BCAA) [11].

In the guideline of American Diabetes Association (ADA), the recommendation of nutritional treatment for patients with diabetes would be based on abilities and adequate goals. It also includes the preferences and individualized healthy diet for each patient. With these related factors, applicable eating patterns have to be considered consisting of nutrientdense and high quality meals. The influencing factors include body weight, blood glucose, blood pressure, lipid profile and diabetic complications [4].

Several recommended health eating patterns are the Mediterranean diet, dietary approaches to stop hypertension (DASH) [12,13], Mediterranean-DASH intervention for neurodegenerative delay (MIND), LCD and plant-based diet (PBD) [14]. PBD is a regimen which encourages whole plant-based foods and discourages meats, dairy products, eggs or all processed/refined foods [15]. PBD was acknowledged as appropriate in medical nutrition therapy for T2DM by the Canadian Diabetes Association (CDA) [16].

For the elderly diabetic patients, there are some other recommendations on the nutrient requirements for the daily meals, such as fibers and liquids. Fiber intake is advised as 25-35 g per day, which is a little more than before. However, we pay attention to increasing fiber in the patients with delayed gastric emptying (gastroparesis). Encouraging fluid intake has been important for preventing constipation, which is often found for older diabetics [17].

One of the most common problem in elder people is the fluid and electrolyte disturbance. Among them, dehydration has to be always prevented [17]. Especially, patients on anti-hypertensives and diuretics are avoided from fluid and electrolyte depletion [18]. We can reduce such episode and its prevalence by various interventions associated with careful attention [19].

There are some elderly diabetic patients, who cannot achieve good glycemic control by lifestyle modification. In such cases, management would be not strictly continued, but loosely changed for the situation of the patients. We can consider social circumstances, living in home or nursing home, psychological status and can adapt it adequately.

For elderly residents in nursing home, some diabetic patients can maintain stable glucose variability with not diabetic formula but rather regular diet [20]. In some reports, tailored diet to personal preferences, culture and goals can make QOL and ADL better, leading to the daily satisfaction with nutritional status [20].

When treating diabetic patients, adequate management has been different in young or older cases. For community-dwelling elderly diabetics, nutrient-dense diet or protein-rich meal has been indispensable for maintaining health and preventing nutritional problems [21]. Due to some evidence, restricted daily diets could impose increased risks for frailty, sarcopenia and malnutrition [22]. Consequently, we should judge and obtain satisfactory management for elderly diabetic patients with the integration of $L C D$ and $C R$, as well as POR and PCM.

In summary, diabetic patients are necessary to have adequate nutrition treatment such as LCD and CR. For elderly diabetic patients, not only calorie/carbohydrate but also protein intake should be considered. Based on POR and PCM, judgment can be determined on the axis of bio-psycho-social concept in primary care. The goal can be set to the adequate management contributing to the patient's satisfactory life.

\section{References}

1. Sacristán JA (2013) Patient-centered medicine and patientoriented research: Improving health outcomes for individual patients. BMC Med Inform Decis Mak 13: 6.

2. Ebe K, Bando H, Yamamoto K, et al. (2018) Daily carbohydrate intake correlates with $\mathrm{HbA} 1 \mathrm{c}$ in low carbohydrate diet (LCD). J Diabetol 1: 4-9.

3. Bando H, Ebe K, Muneta T, et al. (2018) Evaluating pancreas function by meal tolerance test (MTT) in diabetes. American Journal of Diabetes Research 1: 101-109.

4. American Diabetes Association (2019) 5. Lifestyle management: Standards of medical care in diabetes-2019. Diabetes Care 42: S46-S60.

5. Inzucchi SE, Bergenstal RM, Buse JB, et al. (2015) Management of hyperglycemia in type 2 diabetes, 2015: A patient centered approach: Update to a position statement of the American Diabetes Association and the European Association for the Study of Diabetes. Diabetes Care 38: 140-149.

6. Weinger K, Beverly EA, Smaldone A (2014) Diabetes self-care and the older adult. West J Nurs Res 36: 1272-1298.

7. Joaquín C, Puig R, Gastelurrutia $P$, et al. (2018) Mini nutritional assessment is a better predictor of mortality than subjective global assessment in heart failure out-patients. Clin Nutr. 

3(1):49-51

8. Cascio BL, Logomarsino JV (2018) Evaluating the effectiveness of five screening tools used to identify malnutrition risk in hospitalized elderly: A systematic review. Geriatr Nurs 39: 95-102.

9. Leon-Sanz M, Brosa M, Planas M, et al. (2015) PREDyCES study: The cost of hospital malnutrition in Spain. Nutrition 31: 10961102.

10. Op den Kamp CM, Langen RC, Haegens A, et al. (2009) Muscle atrophy in cachexia: Can dietary protein tip the balance? Curr Opin Clin Nutr Metab Care 12: 611-616.

11. Baldwin C, Spiro A, Ahern R, et al. (2012) Oral nutritional interventions in malnourished patients with cancer: A systematic review and meta-analysis. J Natl Cancer Inst 104: 371-385.

12. Cespedes EM, Hu FB, Tinker L, et al. (2016) Multiple healthful dietary patterns and type 2 diabetes in the women's health initiative. Am J Epidemiol 183: 622-633.

13. Ley SH, Hamdy O, Mohan V, et al. (2014) Prevention and management of type 2 diabetes: Dietary components and nutritional strategies. Lancet 383: 1999-2007.

14. Rinaldi S, Campbell EE, Fournier J, et al. (2016) Comprehensive review of the literature supporting recommendations from the Canadian Diabetes Association for the use of a plant-based diet for management of type 2 diabetes. Can J Diabetes 40: 471-477.

15. Lee V, McKay T, Ardern Cl (2015) Awareness and perception of plant-based diets for the treatment and management of type 2 diabetes in a community education clinic: A pilot study. J Nutr Metab 2015.

16. Dworatzek PD, Arcudi K, Gougeon R, et al. (2013) Nutrition therapy. Can J Diabetes 37: S45-S55.

17. Hooper L, Bunn D, Jimoh FO, et al. (2014) Water-loss dehydration and aging. Mech Ageing Dev 137: 50-58.

18. Keller H, Beck AM, Namasivayam A, et al. (2015) Improving food and fluid intake for older adults living in long-term care: $A$ research agenda. J Am Med Dir Assoc 16: 93-100.

19. Bunn D, Jimoh F, Wilsher SH, et al. (2015) Increasing fluid intake and reducing dehydration risk in older people living in long-term care: A systematic review. J Am Med Dir Assoc 16: 101-113.

20. Munshi MN, Florez H, Huang ES, et al. (2016) Management of diabetes in long-term care and skilled nursing facilities: A position statement of the American Diabetes Association. Diabetes Care 39: 308-318.

21. Bernstein $M$ (2017) Nutritional needs of the older adult. Phys Med Rehabil Clin N Am 28: 747-766.

22. Biolo G, Cederholm T, Muscaritoli M (2014) Muscle contractile and metabolic dysfunction is a common feature of sarcopenia of aging and chronic diseases: From sarcopenic obesity to cachexia. Clin Nutr 33: 737-748. 